\title{
SUPERCONDUCTIVITY OF THE WEAK HOPPING ANDERSON LATTICE WITH GENERALIZED ELECTRON INTERACTIONS
}

\author{
DUONg HaI TRIEU \\ Instytut Informatyki, Akademia Górniczo-Hutnicza \\ Al. A. Mickiewicza 30, 30-059 Kraków, Poland* \\ (Received October 30,1990; in final form August 2, 1991)
}

\begin{abstract}
We study the Anderson lattice with a weak hopping amplitude $t$ and generalized electron interactions. The interactions between $f$ electrons, between $f$ and conduction electrons and between conduction electrons are taken into account. An effective Hamiltonian in a second order perturbation in $t$ is derived. Using this Hamiltonian, we study superconductivity. Singlet $d$-like superconductivity may occur in presence of the above interactions. In some cases the interactions lead to suppressing superconductivity.
\end{abstract}

PACS numbers: 74.20.-z

\section{Introduction}

The purely electronic mechanism of superconductivity is an attractive problem. Bastide and Lacroix $[1,2]$ have given a convincing proof of the possibility of this mechanism in the Anderson lattice model with a weak hopping parameter $t$. In this work the authors have only taken into account the infinite interaction between $f$ electrons. However, in fact the interactions between conduction electrons and between conduction and $f$ electrons may be strong in comparison with other parameters of the problem. In this situation, it is desirable to consider all the parameters while resolving the problem.

We consider the Anderson lattice Hamiltonian of the form:

*Present address: Bo mon Vatly, Dai hoc Mo-Dia chat, Tu liem, Hanoi, Vietnam. 


$$
\begin{aligned}
& H=E_{0} \sum_{i \sigma} f_{i \sigma}^{+} f_{i \sigma}+V \sum_{i \sigma}\left(c_{i \sigma}^{+} f_{i \sigma}+f_{i \sigma}^{+} c_{i \sigma}\right)+U_{f} \sum_{i} n_{f i_{1}} n_{f i_{1}} \\
& +U_{c} \sum_{i}^{i \sigma} n_{c i_{1}} n_{c i i_{1}}+U_{f c} \sum_{i}^{i \sigma} n_{f i} n_{c i}-J_{f c} \sum_{i \sigma \sigma^{\prime}} f_{i \sigma}^{+} f_{i \sigma^{\prime}} c_{i \sigma^{\prime}}^{+} c_{i \sigma}+t \sum_{i j \sigma} c_{i \sigma}^{+} c_{j \sigma} .
\end{aligned}
$$

Here, besides the quantities appearing in Ref: [1] we have introduced new parameters: $U_{c}$ - the interaction between conduction electrons, $U_{f c}$ - the classic repulsion between conduction and $f$ electrons and $J_{f c}-$ the parameter of the $f-c$ exchange interaction. The expression of $H$ can be derived, for example from Ref. [3], for a lattice with a non-degenerated $f$-orbital and a non-degenerated $c$-orbital on each site. As in Ref. [1] $U_{f}$ is taken to be infinite. In Sec. 2 we will find the atomic eigenstates. In Sec. 3 we will use these states to construct an effective Hamiltonian. Basing on this Hamiltonian we will study the possibility of superconductivity in Sec. 4.

\section{The atomic states}

There are two one-electron states $|A \sigma\rangle$ with lower energy $E_{A}$ and $|B \sigma\rangle$ with higher energy $E_{B}$, for each spin $\sigma$. These states and their energies remain the same as in [1]. For a site with two electrons there are two singlets and a triplet. The form of these states is the same as in [1] but the expressions for $\cos \varphi$ and $\sin \varphi$ in [1] must be replaced respectively by

$$
\begin{aligned}
& \cos \varphi=\frac{1}{\sqrt{2}}\left\{1-\frac{E_{0}+U_{f c}+J_{f c}-U_{c}}{\left[\left(E_{0}+U_{f c}+J_{f c}-U_{c}\right)^{2}+8 V^{2}\right]^{1 / 2}}\right\}^{1 / 2}, \\
& \sin \varphi=\frac{1}{\sqrt{2}}\left\{1+\frac{E_{0}+U_{f c}+J_{f c}-U_{c}}{\left[\left(E_{0}+U_{f c}+J_{f c}-U_{c}\right)^{2}+8 V^{2}\right]^{1 / 2}}\right\}^{1 / 2} .
\end{aligned}
$$

The energies of the singlets and the triplet are respectively

$$
\begin{aligned}
& E_{S_{1}, S_{2}}=\left\{E_{0}+U_{f c}+J_{f c}+U_{c} \mp\left[\left(E_{0}+U_{f c}+J_{f c}-U_{c}\right)^{2}+8 V^{2}\right]^{1 / 2}\right\} / 2,(4) \\
& E_{T}=E_{0}+U_{f c}-J_{f c} .
\end{aligned}
$$

There is one three-electron state $|C \sigma\rangle$ for each spin. The expression for the state is the same as in [1] and its energy is

$E_{C}=E_{0}+U_{c}+2 U_{f c}-J_{f c}$.

\section{Effective Hamiltonian}

Using a perturbation technique $[1,4]$ we can construct an effective Hamiltonian which acts on the subspace of degenerated ground states $\Omega$. In our approach, the operator $T$ plays the role of a perturbation. The results depend on the mean number of electrons per site $n$. We will consider three possible cases: $0<n<1$, $1<n<2$ and $2<n<3$. 


\subsection{The case $0<n<1$}

If $U_{f c}, J_{f c}$ and $U_{c}$ are equal to zero or small, the unperturbed ground state is a mixture of sites with an electron on the state $|A \sigma\rangle$ and unoccupied sites. It is so when

$$
\begin{aligned}
& E_{S_{1}}-2 E_{A} \gg|t|, \\
& E_{T}-E_{S_{1}} \gg|t|, \\
& E_{A}+E_{C}-2 E_{S_{1}} \gg|t| .
\end{aligned}
$$

These conditions are also needed for the validity of the perturbation expansion. They restrict the region of values of $U_{f c}, J_{f c}$ and $U_{c}$. The cases when the conditions (7-9) are not satisfied need a further investigation. We will show by numerical calculations that these conditions are satisfied for almost all typical values of the interaction parameters.

Performing analogous calculations as in [1], we obtain the effective Hamiltonian $\tilde{H}=\tilde{H}_{1}+\tilde{H}_{2 a}+\tilde{H}_{2 b}$, where $\tilde{H}_{1}$ is the first-order effective Hamiltonian, $\tilde{H}_{2 a}$ is the second-order two-site effective Hamiltonian and $\tilde{H}_{2 b}$ is the second-order three-site effective Hamiltonian. The expression for $\tilde{H}_{1}$ is of the form:

$$
\tilde{H}_{1}=t_{1} \sum_{i j \sigma} a_{i \sigma}^{+} a_{j \sigma}+U \sum_{i} n_{i_{1}} n_{i_{1}}
$$

where $t_{1}=t \sin ^{2} \theta, a_{i \sigma}^{+}$is the operator which creates an electron in the state $|A \sigma\rangle$ on site $i: a_{i \sigma}^{+}|i 0\rangle=|i A \sigma\rangle$ and $n_{i \sigma}=a_{i \sigma}^{+} a_{i \sigma}$. Here we have introduced the parameter $U=\infty$ to restrict our system to the subspace $\Omega$. The expressions for $\tilde{H}_{2 a}$ and $\tilde{H}_{2 b}$ are

$$
\begin{aligned}
& \tilde{H}_{2 a}=\frac{g}{2} \sum_{i j} n_{i} n_{j}-\frac{I}{2} \sum_{i j} S_{i} \cdot S_{j}, \\
& \tilde{H}_{2 b}=t_{2} \sum_{i j l \sigma} a_{i \sigma}^{+} a_{l \sigma}+g^{\prime} \sum_{i j l \sigma} a_{i \sigma}^{+} a_{l \sigma} n_{j}-I^{\prime} \sum_{i j l} S_{i l} \cdot S_{j} .
\end{aligned}
$$

Here $i$ and $j$ as well as $j$ and $l$ are nearest-neighbours, $n_{i}=\sum_{\sigma} a_{i \sigma}^{+} a_{i \sigma}, S_{i}$ is the spin operator: $S_{i}^{z}=\left(a_{i_{1}}^{+} a_{i_{1}}-a_{i_{1}}^{+} a_{i_{1}}\right) / 2, S_{i}^{+}=a_{i_{1}}^{+} a_{i_{1}}, S_{i}^{-}=a_{i_{1}}^{+} a_{i_{1}}$ and the operator $S_{i l}$ is defined as: $S_{i l}^{z}=\left(a_{i_{1}}^{+} a_{l_{1}}-a_{i_{1}}^{+} a_{l_{1}}\right) / 2, S_{i l}^{+}=a_{i_{1}}^{+} a_{l_{l}}, S_{i l}^{-}=a_{i_{1}}^{+} a_{l_{\mathrm{f}}}$. The parameters in the expressions for $\tilde{H}_{2 a}$ and $\tilde{H}_{2 b}$ are

$$
\begin{aligned}
& I=-\frac{4 t^{2} \sin ^{2} \theta}{E_{S_{1}}-2 E_{A}}\left(\sin \varphi \sin \theta+\frac{\cos \varphi \cos \theta}{\sqrt{2}}\right)^{2} \\
& -\frac{4 t^{2} \sin ^{2} \theta}{E_{S_{2}}-2 E_{A}}\left(\cos \varphi \sin \theta-\frac{\sin \varphi \cos \theta}{\sqrt{2}}\right)^{2}+\frac{2 t^{2} \sin ^{2} \theta \cos ^{2} \theta}{E_{T}-2 E_{A}} \\
& g=I / 4+\frac{2 t^{2} \sin ^{2} \theta \cos ^{2} \theta\left(U_{f c}-J_{f c}\right)}{\left[E_{0}^{2}+4 V^{2}\right]^{1 / 2}\left[U_{f c}-J_{f c}+\left(E_{0}^{2}+4 V^{2}\right)^{1 / 2}\right]}
\end{aligned}
$$




$$
\begin{aligned}
& t_{2}=-\frac{t^{2} \cos ^{2} \theta \sin ^{2} \theta}{E_{B}-E_{A}}, \\
& g^{\prime}=g / 2, \\
& I^{\prime}=I / 2 .
\end{aligned}
$$

\subsection{The case $1<n<2$}

If the conditions (7-9) are satisfied in the ground state electrons occupy the states $|A \sigma\rangle$ and $\left|S_{1}\right\rangle$. The form of the effective Hamiltonian remains the same as above if we introduce a new operator $a_{i \sigma}^{+}$which creates a particle $A \sigma$ on a background of singlets $S_{1}: a_{i \sigma}^{+}\left|i S_{1}\right\rangle=|i A \sigma\rangle$. The state completely filled by singlets $S_{1}$ on each site now plays the role of vacuum. The parameters of $\tilde{H}$ now have the form:

$$
\begin{aligned}
& t_{1}=-(t / 2)(\cos \theta \cos \varphi+\sqrt{2} \sin \theta \sin \varphi)^{2} \\
& g=2\left(f_{1}+f_{2}+f_{3}+f_{4}+3 f_{5}+3 f_{6}\right)-(3 / 2) f_{7}-f_{8}-f_{9}-2\left(f_{10}+f_{11}\right) \\
& I=2 f_{7}-4 f_{8}-4 f_{9} \\
& t_{2}=-f_{2}+f_{10} / 2 \\
& g^{\prime}=f_{2}+\left(f_{3}+3 f_{5}-f_{8}-f_{10}\right) / 2 \\
& I^{\prime}=2\left(-f_{3}+f_{5}-f_{8}\right)
\end{aligned}
$$

where

$$
\begin{aligned}
& f_{1}=\frac{t^{2} \cos ^{2} \varphi \sin ^{2} \theta}{2\left(E_{C}-E_{A}-E_{S_{1}}\right)} \\
& f_{2}=\frac{t^{2}}{E_{B}-E_{A}}\left(\sin \varphi \sin \theta+\frac{\cos \varphi \cos \theta}{\sqrt{2}}\right)^{2} \\
& \times\left(-\sin \varphi \cos \theta+\frac{\cos \varphi \sin \theta}{\sqrt{2}}\right)^{2} \\
& f_{3}=\frac{t^{2}}{E_{S_{2}}-E_{S_{1}}}\left(\cos \varphi \sin \theta+\frac{\sin \varphi \cos \theta}{\sqrt{2}}\right)^{2} \\
& \times\left(\sin \varphi \sin \theta+\frac{\cos \varphi \cos \theta}{\sqrt{2}}\right)^{2}, \\
& f_{4}=\frac{t^{2}(\cos \varphi \sin \theta-\sin \varphi \cos \theta / \sqrt{2})^{2}(-\sin \varphi \cos \theta+\cos \varphi \sin \theta / \sqrt{2})^{2}}{E_{S_{2}}-E_{S_{1}}+E_{B}-E_{A}},
\end{aligned}
$$




$$
\begin{aligned}
f_{5} & =\frac{t^{2} \cos ^{2} \theta}{2\left(E_{T}-E_{S_{1}}\right)}(\sin \varphi \sin \theta+\cos \varphi \cos \theta / \sqrt{2})^{2}, \\
f_{6} & =\frac{t^{2} \cos ^{2} \theta}{2\left(E_{B}-E_{A}+E_{T}-E_{S_{1}}\right)}(\sin \varphi \cos \theta-\cos \varphi \sin \theta / \sqrt{2})^{2}, \\
f_{7} & =\frac{t^{2} \sin ^{2} \theta \cos ^{2} \theta}{E_{T}-2 E_{A}} \\
f_{8} & =\frac{t^{2} \sin ^{2} \theta}{\left(E_{S_{1}}-2 E_{A}\right)}(\sin \varphi \sin \theta+\cos \varphi \cos \theta / \sqrt{2})^{2}, \\
f_{9} & =\frac{t^{2} \sin ^{2} \theta}{\left(E_{S_{2}}-2 E_{A}\right)}(\cos \varphi \sin \theta-\sin \varphi \cos \theta / \sqrt{2})^{2}, \\
f_{10} & =\frac{t^{2} \cos ^{2} \varphi}{\left(E_{C}+E_{A}-2 E_{S_{1}}\right)}(\sin \theta \sin \varphi+\cos \theta \cos \varphi / \sqrt{2})^{2}, \\
f_{11} & =\frac{t^{2} \cos ^{2} \varphi}{\left(E_{C}+E_{B}-2 E_{S_{1}}\right)}(\cos \theta \sin \varphi-\sin \theta \cos \varphi / \sqrt{2})^{2} .
\end{aligned}
$$

\subsection{The case $2<n<3$}

If the conditions (7-9) are satisfied, the ground state is a mixture of sites with three electrons in the state $\mid C \sigma)$ and sites with two electrons in the state $\left|S_{1}\right\rangle$. We obtain again the same expressions for the effective Hamiltonian as above if we introduce a new operator $a_{i \sigma}^{+}$which creates a particle $C \sigma$ on the background $S_{1}: a_{i o}^{+}\left|i S_{1}\right\rangle=|i C \sigma\rangle$. The expressions for the parameters now have the form:

$$
\begin{aligned}
& t_{1}=\frac{t \cos ^{2} \varphi}{2} \\
& g=\frac{1}{2}\left(f_{12}+3 f_{13}\right)-2\left(f_{10}+f_{11}\right), \\
& I=0, \\
& t_{2}=\frac{1}{2}\left(f_{10}+f_{11}\right), \\
& g^{\prime}=g / 4, \\
& I^{\prime}=\frac{1}{2}\left(-f_{12}+f_{13}\right),
\end{aligned}
$$

where

$$
\begin{aligned}
& f_{12}=\frac{t^{2} \cos ^{2} \varphi \sin ^{2} \varphi}{E_{S_{2}}-E_{S_{1}}} \\
& f_{13}=\frac{t^{2} \cos ^{2} \varphi}{E_{T}-E_{s_{1}}}
\end{aligned}
$$




\section{Superconductivity}

The effective Hamiltonian derived above can be used to study superconductivity. For this purpose one first transforms the Hamiltonian to the Bloch representation. The terms with $t_{1}$ and $t_{2}$ contribute to the kinetic energy. Retaining only the terms responsible for superconductivity one can reduce the interaction part $\tilde{H}_{2}-t_{2} \sum a_{a \sigma}^{+} a_{l \sigma} \equiv G$ of $\tilde{H}_{2}$ to the BCS extended form $[1,5]$ :

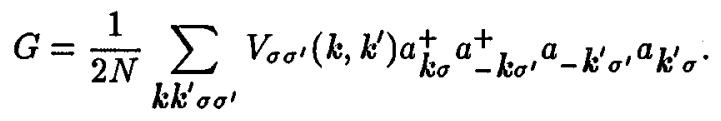

Among various terms of the expression for $G$ the terms with $V_{\sigma \sigma^{\prime}}^{\text {odd }}\left(k, k^{\prime}\right)$ is responsible for triplet superconductivity and the terms with $V_{\sigma,-\sigma}^{\text {even }}\left(k, k^{\prime}\right)$ is responsible for singlet superconductivity. Here the index odd and even respectively mean the odd and even parts with respect to $k$ and $k^{\prime} . V_{\sigma \sigma^{\prime}}^{\text {odd }}\left(k, k^{\prime}\right)$ is independent of spin and can be written as:

$$
V^{\text {odd }}\left(k, k^{\prime}\right)=2 V_{t} \sum_{\alpha} \sin k_{\alpha} \sin k_{\alpha}^{\prime},
$$

where

$$
V_{t}=g-I / 4-2\left(g^{\prime}-I^{\prime} / 4\right) .
$$

$V_{\uparrow \downarrow}^{\text {even }}\left(k, k^{\prime}\right)=V_{\downarrow \uparrow}^{\text {even }}\left(k, k^{\prime}\right)$ can be decomposed into two parts responsible for singlet $s$-like and singlet $d$-like superconductivity:

$$
V_{\uparrow \downarrow}^{\text {even }}\left(k, k^{\prime}\right)=2 V_{s} \beta_{k} \beta_{k^{\prime}} / 3+2 V_{d}\left(\eta_{k} \eta_{k^{\prime}} / 2+\zeta_{k} \zeta_{k^{\prime}} / 6\right),
$$

where

$$
\begin{aligned}
& \beta_{k}=\sum_{\alpha} \cos k_{\alpha}, \\
& \eta_{k}=\cos k_{x}-\cos k_{y}, \\
& \zeta_{k}=\cos k_{x}+\cos k_{y}-2 \cos k_{z}, \\
& V_{s}=g+3 I / 4+10\left(g^{\prime}+3 I^{\prime} / 4\right), \\
& V_{d}=g+3 I / 4-2\left(g^{\prime}+3 I^{\prime} / 4\right) .
\end{aligned}
$$

For the investigation of the possibility of superconductivity we calculate the coupling constants in three channels: $V_{s}$ for the singlet $s$-like channel, $V_{d}$ for the singlet $d$-like channel and $V_{t}$ for the triplet channel. 


\subsection{The case $0<n<1$}

We find

$$
\begin{aligned}
& V_{s}=6 I+\frac{12 t^{2} \sin ^{2} \theta \cos ^{2} \theta\left(U_{f c}-J_{f c}\right)}{\left(E_{0}^{2}+4 V^{2}\right)^{1 / 2}\left[U_{f c}-J_{f c}+\left(E_{0}^{2}+4 V^{2}\right)^{1 / 2}\right]}, \\
& V_{d}=0, \\
& V_{t}=0 .
\end{aligned}
$$

The detail investigation of $V_{s}$ shows that it may be negative. The interactions of large value may lead to $V_{s}>0$ and suppress superconductivity. For example, $V_{s} \approx 3 t^{2} / 2 V>0$ for $E_{0}=J_{f c}=0, U_{c} \gg V$ and $U_{f c} \gg V$, at the same time $V_{s}=-27 t^{2} / 2 V<0$ for $E_{0}=J_{f c}=U_{c}=U_{f c}=0$. We note that $V_{s}>0$ is a sufficient condition for the absence of singlet $s$-like superconductivity, at the same time $V_{s}<0$ is a necessary but not sufficient condition for the presence of this superconductivity because this superconductivity is also sensitive to the on-site repulsion [1]. So if $V_{s}<0$ one still cannot conclude definitely that singlet $s$-like superconductivity is present. Because of this ambiguity we will not investigate the $s$-like superconductivity in the following.

\subsection{The case $1<n<2$}

Some interesting informations can be obtained when studying the limit cases $E_{0} / V \ll-1, E_{0} / V=0$ and $E_{0} / V \gg 1$.

\subsubsection{The case $E_{0} / V \ll-1, \quad 1<n<2$}

On the assumption that $\left|E_{0}\right| \gg U_{c}, U_{f c},\left|J_{f c}\right|$ we obtain

$$
\begin{aligned}
& V_{d} \approx-\frac{t^{2}}{6\left(U_{c} / 3-J_{f c}-V^{2} / E_{0}\right)}<0, \\
& V_{t} \approx-\frac{t^{2}}{2}\left(\frac{1}{J_{f c}+V^{2} / E_{0}}-\frac{1}{3 J_{f c}+3 V^{2} / E_{0}-U_{c}}\right)>0 .
\end{aligned}
$$

Here we have used the conditions (7-9). Because $V_{d}<0$, superconductivity may occur in the singlet $d$-like channel [1]. The infinite on-site repulsion does not affect the existence of this superconductivity because the pairing occurs only for particles on neighbouring sites. We note that the absolute value of $V_{d}$ is reduced in the presence of the interactions.

\subsubsection{The case $E_{0} / V=0, \quad 1<n<2$}

If $U_{f c}=J_{f c}=U_{c}=0$ we obtain the results of Ref. [1]. The small values of $U_{f c}, J_{f c}$ and $U_{c}$ may change a little the quantities $V_{d}$ and $V_{t}$ but the main conclusions of Ref. [1] remain. Because of this we concentrate on the cases of large values of the interaction parameters. In fact, among $U_{f c}, J_{f c}$ and $U_{c}, J_{f c}$ is the 
smallest and $U_{f c}$ is the largest. We will study the case of very large value of $U_{f c}$ : $U_{f c} \gg U_{c}, J_{f c}, V, t$.

Using this condition and the conditions (7-9) we obtain

$$
V_{d} \approx V_{t} \approx \frac{t^{2}\left(2 U_{f c}+U_{C}\right)}{2 U_{f c}\left(U_{f c}-U_{c}\right)}>0 \text {. }
$$

\subsubsection{The case $E_{0} / V \gg 1, \quad 1<n<2$}

If $E_{0} / U_{f c} \gg 1, E_{0} / U_{c} \gg 1$ and $E_{0} /\left|J_{f c}\right| \gg 1$ the conditions (7-9) are satisfied automatically. The coupling constants are small, $V_{d}=0\left(t^{2} / E_{0}\right)$ and $V_{t}=$ $0\left(t^{2} / E_{0}\right)$.

\subsection{The case $2<n<3$}

\subsubsection{The case $E_{0} / V \ll-1,2<n<3$}

In this case the values of $V_{d}, V_{t}$ are the same as in the case $1<n<2$, $E_{0} / V \ll-1$.

4.3.2. The case $E_{0} / V=0, \quad 2<n<3$

$U_{f c} \gg U_{c}, J_{f c}, V, t$, the coupling constants are small, $V_{d}=0\left(t^{2} / U_{f c}\right)$ and $V_{t}=0\left(t^{2} / U_{f c}\right)$.

\subsubsection{The case $E_{0} / V \gg 1, \quad 2<n<3$}

As in [1] the coupling constants are small, $V_{d}=0\left(t^{2} V^{2} / E_{0}^{3}\right)$ and $V_{t}=$ $0\left(t^{2} V^{2} / E_{0}^{3}\right)$.

\subsection{Numerical calculations}

As an illustration we calculate $V_{d}$ and $V_{t}$ for several values of the interaction parameters. We assume that the model describes superconductivity in some heavy-fermion rare-earth and actinide systems. From [6] we have some orientation values of the parameters of a rare-earth system: $U_{f} \approx 100 \mathrm{eV}, U_{c} \approx 2 \mathrm{eV}, J_{f c} \approx$ $-0.2 \div 0.2 \mathrm{eV}, E_{0} \approx-2.5 \mathrm{eV}, V \approx 0.15 \div 0.55 \mathrm{eV}$. The parameter $t$ may vary in a large extent, so we will not take a concrete value of $t$ but express the final rsults in $t$ suggesting it is sufficiently small. The value of $U_{f c}$ is not given in [6] but from the physical consideration $U_{f c}$ seems to be large than $U_{c}$. The results of calculations of $V_{d}$ for $J_{f c}=0, U_{c}=0 \div 2 \mathrm{eV}, U_{f c}=0 \div 8 \mathrm{eV}$ are listed in Table I (for $1<n<2$ ) and Table II (for $2<n<3$ ). The dependence of $V_{d}$ on $J_{f c}$ is illustrated in Table III, where we have taken for $U_{c}$ and $U_{f c}$ small values $\left(U_{c}=1 \mathrm{eV}\right.$ and $\left.U_{f c}=2 \mathrm{eV}\right)$ to guarantee superconductivity. In these tables the values of $U_{f}, E_{0}$ and $V$ are equal to $\infty,-2.5 \mathrm{eV}$ and $0.4 \mathrm{eV}$, respectively. For all values used in Tables I, II III conditions (7-9) are satisfied. We note that in Table III we have taken for $J_{f c}$ a small value when $J_{f c}>0$. For a large positive value of $J_{f c}$ conditions (7-9) may be not satisfied. For example $J_{f c}=0.1 \mathrm{eV}$ (and $\left.U_{c}=1 \mathrm{eV}, U_{f c}=2 \mathrm{eV}, E_{0}=-2.5 \mathrm{eV}, V=0.4 \mathrm{eV}\right)$ we have $E_{T}-E_{S_{1}}<0$. 


\section{TABLE I}

\begin{tabular}{c|c|c|c|c}
\multicolumn{5}{c}{$V_{d} / t^{2}\left[\mathrm{eV}^{-1}\right]$ for $1<n<2}$. \\
\hline \hline \multirow{2}{*}{$U_{c}[\mathrm{eV}]$} & \multicolumn{4}{|c}{$U_{f c}[\mathrm{eV}]$} \\
\cline { 2 - 5 } & 0 & 2 & 4 & 8 \\
\hline 0 & -2.680 & -0.255 & 0.761 & 0.455 \\
1 & -0.437 & -0.310 & 0.686 & 0.514 \\
2 & -0.233 & -0.214 & 0.125 & 0.588
\end{tabular}

TABLE II

\begin{tabular}{c|r|c|c|c}
\multicolumn{5}{c}{$V_{d} / t^{2}\left[\mathrm{eV}^{-1}\right.$ for $2<n<3}$. \\
\hline \hline \multirow{2}{*}{$U_{c}[\mathrm{eV}]$} & \multicolumn{4}{|c}{$U_{f c}[\mathrm{eV}]$} \\
\cline { 2 - 5 } & 0 & 2 & 4 & 8 \\
\hline 0 & 2.681 & -0.340 & 0.030 & 0.001 \\
1 & -0.440 & -0.308 & 0.107 & 0.002 \\
2 & -0.237 & -0.211 & 0.025 & 0.004
\end{tabular}

Dependence of $V_{d} / t^{2}$ on $J_{f c}$.

TABLE III

\begin{tabular}{c|c|c}
\hline \hline$J_{f c}[\mathrm{eV}]$ & $V_{d} / t^{2}\left[\mathrm{eV}^{-1}\right]$ for $1<n<2$ & $V_{d} / t^{2}\left[\mathrm{eV}^{-1}\right]$ for $2<n<3$ \\
\hline-0.2 & -0.255 & -0.217 \\
-0.1 & -0.260 & -0.254 \\
0 & -0.310 & -0.308 \\
0.05 & -0.344 & -0.344
\end{tabular}

As it can be seen from Tables I and II, singlet $d$-like superconductivity is still possible for small values of the interaction parameters, but the absolute value of $V_{d}$ is reduced by the interactions $U_{c}$ and $U_{f c}$. For large values of $U_{c}$ and $U_{f c}$ (with $U_{f c}>U_{c}$ ) $V_{d}$ is positive and this superconductivity is suppressed. From Table III we see that this superconductivity is more favourable for $J_{f c}>0$ than for $J_{f c}<0$. We remind that $J_{f c}>0(<0)$ corresponds to the ferromagnetic (antiferromagnetic) $f-c$ exchange. It can be seen if we express the term with $J_{f c}$ in expression (1) in the form:

$$
-2 J_{f c} \sum_{i} S_{f i} \cdot S_{c i}-\frac{J_{f c}}{2} \sum_{i} n_{f i} n_{c i}
$$

We have also calculated $V_{i}$ for the above values of the parameters. We obtain $V_{i}>0$ and triplet superconductivity is absent.

\section{Conclusions}

We have investigated the weak-hopping Anderson lattice with generalized electron interactions. In the absence of the interactions our results agree with the results of Ref. [1]. We find that singlet $d$-like superconductivity is possible in some 
cases. The absolute value of $V_{d}$ is reduced by the interactions $U_{c}$ and $U_{f c}$. The large interactions $U_{c}$ and $U_{f c}\left(U_{f c}>U_{c}\right)$ lead to positive values of $V_{d}$ and suppress superconductivity. Singlet $d$-like superconductivity is more favourable for $J_{f c}>0$ than for $J_{f c}<0$. Triplet superconductivity has not been found.

\section{Acknowledgements}

This work was supported in part by the Institute of Physics of the Polish Academy of Sciences, problem 01.04.

\section{References}

[1] C. Bastide, C. Lacroix, J. Phys. C, Solid State Phys. 21, 3557 (1988).

[2] C. Bastide, C. Lacroix, Europhys. Lett. 4, 936 (1987).

[3] L.L. Hirst, Adv. Phys. 27, 231 (1987).

[4] M.H.L. Pryce, Proc. Phys. Soc. A 63, 25 (1950).

[5] K. Miyake, T. Matsuura, H. Jichu, Y. Nagaoka, Prog. Theor. Phys. 72, 1063 (1984).

[6] W. Nolting, A. Ramakanth, Phys. Rev. B 33, 1838 (1985). 